\title{
An Automatic System for Detecting the Vehicle Registration Plate from Video in Foggy and Rainy Environments using Restoration Technique
}

\author{
Savneet Kaur \\ M.tech (CSE) \\ GNDEC LUDHIANA
}

\author{
Kamaljit Kaur \\ Dhillon \\ Assistant Professor \\ GNDEC LUDHIANA
}

\author{
Manvi \\ Assistant Professor \\ LLRIET, MOGA
}

\author{
Rajdeep Singh \\ Chauhan \\ Assistant Professor \\ LLRIET, MOGA
}

\begin{abstract}
The vehicle registration plate detection is an emerging area in digital image processing. The registration plates are detected by using the video cameras on different locations for controlling the traffic. The vehicle registration plates could be detected in normal environment, but when the environment changes to foggy or rainy then the working of the system which detects the registration plate of vehicle got effected. This work concentrates to detect the Registration plates (Licensing Plates) of vehicle in such environments using restoration technique. Moreover this system also converts the images to characters which can be manipulated for future.
\end{abstract}

Keywords: Registration plate, Histogram technique, Restoration Technique, Character Recognition

\section{INTRODUCTION}

\subsection{Vehicle Registration Plate}

VRPD (Vehicle registration plate detection) is a video based technique in which vehicles are identified by their registration plate. In real time the cameras situated at traffic lights, toll plaza, border area etc. captures the video of vehicle so that traffic can be moved out in disciplined way and anyone not obeying the rules can be caught by their vehicles registration plate. In the previous work, many techniques such as canny edge detection and morphological operators, sobel edge detector [5], color model etc are used. All the above techniques work when the image is in clear form i.e. at day time, without noise and in the results only the image of registration plate is extracted out. Registration plates are easily readable by human beings as it is having intensity of very high level but when it is captured by camera then many effects like illumination blur background and foreground color etc. creates a problem. For removing such type of noise a multistage algorithm has been used in this paper. In this paper the videos are of day time but in different environment such as foggy and rainy are implemented. In the previous papers only image of registration plate is extracted in normal environment but with this algorithm image and characters both are displayed including various environments.

\subsubsection{Probability of detection of registration plate from Video over single image:}

When images captured by the camera that is positioned at some fix location then the following problems may occur:

(i) The Probability of image accuracy can be less.
(ii)The image captured can be Corrupted or blurred.

(iii)Some movements of vehicles are missed by the camera.

No software can detect the vehicles registration plate if the images are corrupted or blur. Due to such type of problems the need of video processing occurred.

Advantages of video processing are:

(i) Image can get more accurate when it is captured by video camera as it can miss a single movement.

(ii) If one image does not give the desired results then the number of other image can be used to get desired results from the video of vehicle.

For example: In $10 \mathrm{sec}$, video camera produces 240 frames/images, while photo camera captures approximately 6 images. Hence, Probability of detection of number plate from video over images $(\mathrm{PV})$ is [4]:

$\mathrm{PV}=((\mathrm{V}-\mathrm{I}) \div \mathrm{V}) * 100$

$\mathrm{PV}=((240-6) \div 240) * 100=97.5 \%$

V: denotes images extracted from video camera.

I: denotes images extracted from photo camera.

In video, among 240 images approximately 218 images can show the accurate number plate. In case of photo camera, among 6 images approximately 2 images can give accurate results [4].

\subsection{Restoration Technique}

Image restoration technique is used to improve the quality of the image. It is similar to enhancement. It involves modeling of humiliation and applying the inverse process in order to recover the image. The restoration is categorized into degradation model which contains noise and blur. The categories are spatial domain technique; frequency domain technique and wavelet transform technique.

\section{LITERATURE SURVEY}

Hung, K.M. et. al. (2010) [3] presented an instant and realtime mobile vehicle license plate recognition system in an open environment. In this a nonfixed video camera is installed in the car, the system tries to capture the image of the car in 
front and to process instant vehicle license plate detection and recognition. The location of the two barking lights in the captured image was detected. Then set license plate detection region using the probability distribution of the license plate between the two lights. This method can eliminate any environmental interference during the license plate detection and improve the rate of accuracy of license plate detection and recognition. The morphology method Black Top-Hat is also used to enhance the level of separation of the license plate characters. Experiments show that the system can effectively and quickly capture the vehicle image, detect and recognize the license plate whether it is in daytime, night time, clear day, raining day or under complicated environment.

Lazrus, A. et. al. (2011) [5] discussed that License plate location is an important phase in vehicle license plate recognition for intelligent transport systems. They design and implement an efficient method for LPR (License Plate Recognition) of Indian License Plates. This paper presents a robust method of license plate location, segmentation and reorganization of the characters present in the located plate. The images of various vehicles have been acquired manually and converted in to gray-scale images. The segmentation of gray scale image generated by finding edges using Sobel filter for smoothing image is used to reduce the number of connected component and then bwlabel is used to calculate the connected component. Single character is detected by this technique. The results show that the proposed method achieved accuracy of $98 \%$ by optimizing various parameters with higher recognition rate than the traditional methods.

Kaur, H. et. al. (2012) [4] discussed that Road discipline is must due to growing number of vehicles and traffic violators on roads. In order to enhance discipline upon roads, this paper describes an instant and real time mobile vehicle license plate recognition system in an open environment. License plate recognition system plays a major role in monitoring traffic rules and maintaining law enforcement on public roads. Using a fixed camera installed at red lights, toll plaza, border crossing, and petrol station and in parking areas, system capture the videos of the vehicles. Using canny edge detection algorithm and morphological operators, system detects vehicle number plate. Experiment shows that system can effectively capture the vehicle video and detect the license plate whether it has been taken at day time, night time or under complicated environments and show accuracy up to $90 \%$.

Soon, C.K. et. al. (2012) [8] presented the CNPR (Car Number Plate Detection and Recognition) system that is an electronic payment system, traffic surveillance system and as a police enforcement tools. Number plate in Malaysia mostly contains white character with black background color. In this paper the CNPR system is divided into two main phases. Phase I is to extract the car number plate region whereas Phase II will segment the car plate region into single character, which are then recognized by character classifier.

Sharma, P et. al. (2013) [10] describes a computational algorithm for automatic recognition of vehicle registration plate from recorded video. Popular methods involve the use of technologies like computer vision, image precession, artificial neural networks and fuzzy logic systems. Some of these have been implemented, but none so far have been able to guarantee high levels of accuracy. Also, most of these systems work on still images captured from a camera. In this paper the authors obtain frames having image of moving car from the video using motion detection. The region containing the number plate is then identified by the canny edge detection algorithm and Hough transformation Segmentation of the plate is then undertaken, using histogram analysis, to obtain the separate characters. Neural techniques are then applied to recognize the individual characters.

\section{PROBLEM FORMULATION}

The problem with registration plate of the vehicle is as follows:

It is known that registration number of the vehicle is easily readable by human beings as human vision has high level of intensity. But when it is captured by the camera then some amount of noise is added in the video as well as in image. The noise can be due to blur background or foreground color or due to some bad weather conditions such as fog and rain. The speed of the vehicle is also one of the problem to read the vehicles number.

\subsection{Objectives}

1. To detect registration plate in rainy environment.

2. To detect registration plate in Fog environment.

3. To Change the registration plate in characters.

\section{PRESENT WORK}

The main motive of this paper is to detect vehicle registration plate character from video in different environment. The proposed system works as follows:

First of all a video is captured from the camera which is at fixed location. These locations can be toll plaza, traffic lights, border crossing and petrol station etc. The camera will be at the height of 3 feet. The video captured by the camera can be from front or rear of the vehicle which contains its registration plate.

When the video is captured then it is divided into frames with the help of MATLAB operations. In this paper the work has been done on 10 to $15 \mathrm{sec}$ video clips. The $10 \mathrm{sec}$ video clip contains 240 frames/images. There are three types of frames such as I-frames that is an intra frame, P-frame which is constructed with the difference between the current frame and the closest I-frame and last is B-frame that is constructed from the two closest I or P frames. After the video to frame conversion, the next step is to convert these frames into images. The necessity of this conversion arises because as we know that video is also a type of image. But in videos the rate of change of frames is very fast and the whole environment is covered in video. Therefore, the video is converted into images. On these images certain operations are applied for extracting the registration plate. A multi resolution analysis is done for all the frames. Image restoration and Contrast Enhancement is applied on extracted images to detect registration plate. When registration plate is extracted out then unsharp mask filtering is done to remove the noise such as fog and rain. After removing the noise, an output image comes which contain the registration plate of the vehicle. One more step is done in this paper i.e. the operation of character recognition. The characters of the number plate are detected with the help of character recognition technique. This technique is helpful when anyone wants to check the registration plate from the database, then it can be easily searched from database by just copying and pasting the output characters. 


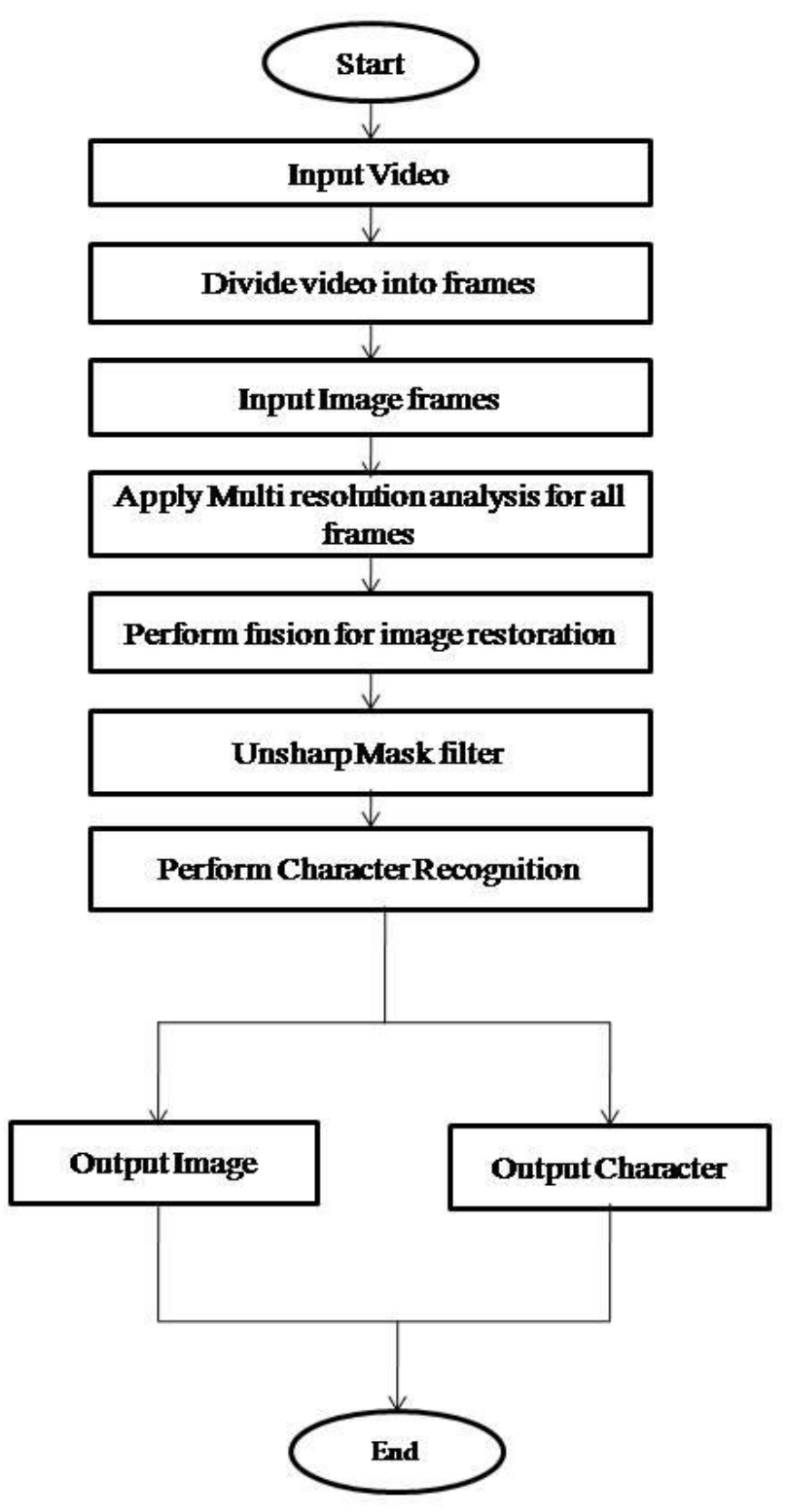

Figure 4.1 Flowchart describing the steps for extracting out the registration plate from video

\section{SIMULATION AND PARAMETERS}

In this work image quality assessment is done with the following:

Table 5.1: Showing the simulation parameters

\begin{tabular}{|l|l|}
\hline Tools & \multicolumn{1}{|c|}{ Parameters } \\
\hline Videos & Videos containing fog and rain \\
\hline Noises & Gaussian and Salt \& pepper \\
\hline Software & MATLAB \\
\hline Version & $2009 \mathrm{~b}$ \\
\hline
\end{tabular}

\section{RESULTS}

\subsection{Vehicle Video with Fog}

The Figure 6.1 shows the video of vehicle that contain noise such as Fog which is considered as Gaussian noise. This is 29 second video from where the fames are extracted and with the help of extracted frames the character of the registration plate are extracted by using various operations in MATLAB. Figure 6.2 shows the extracted character of registration plate from video. 


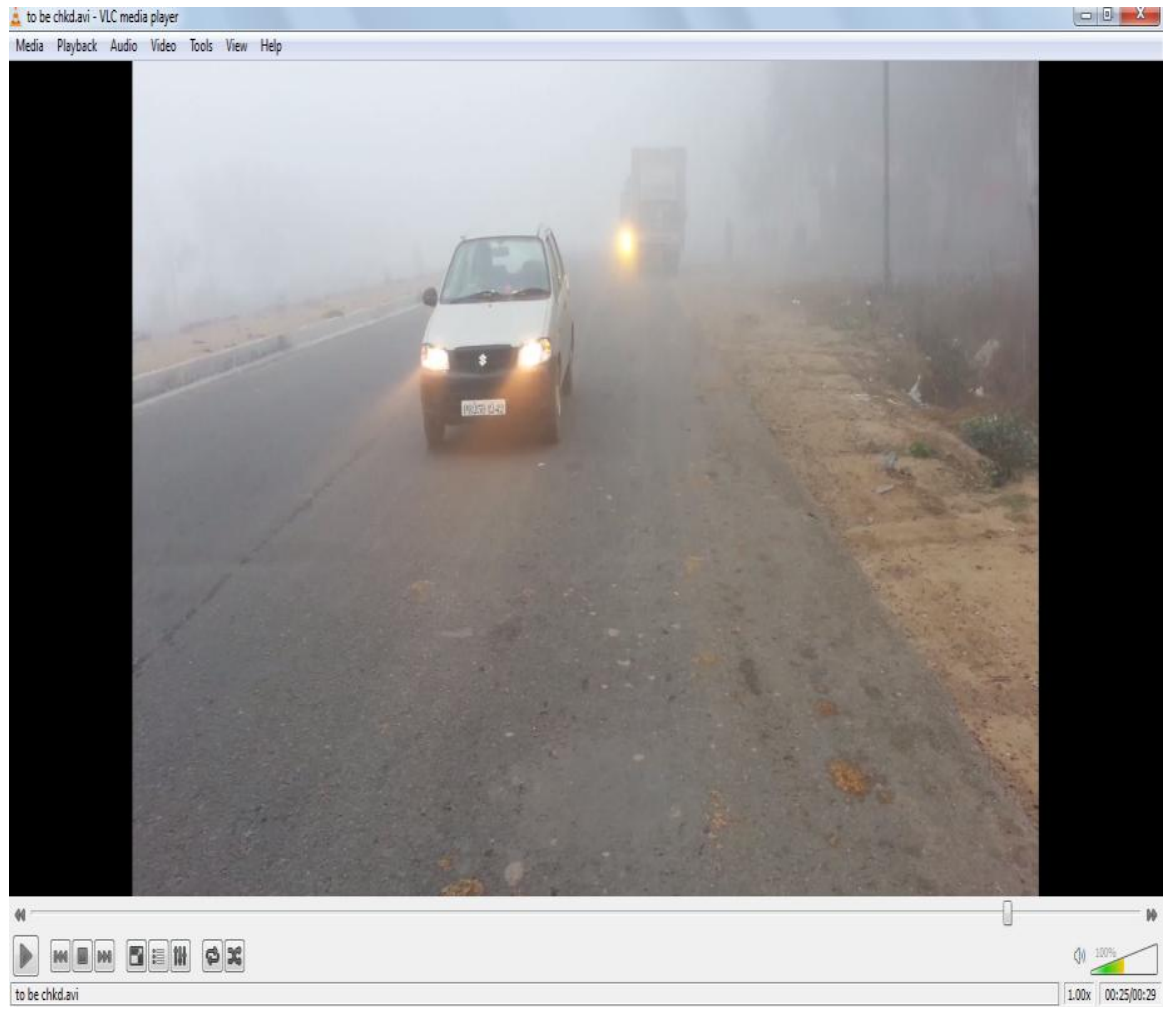

Figure 6.1: Video of vehicle containing Fog

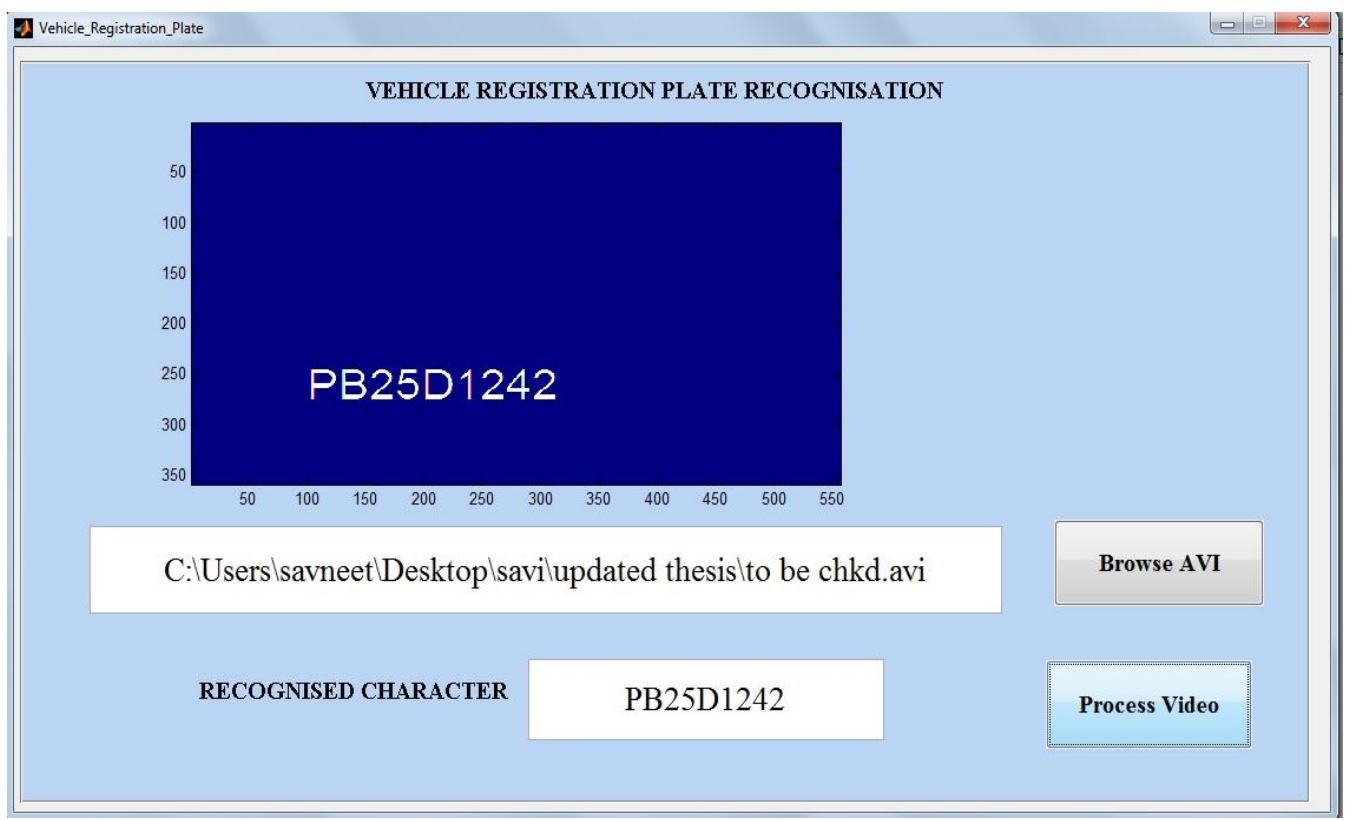

Figure 6.2: Successfully extracted registration plate image and characters from Fog video.

\subsection{Vehicle Video with Rain}

The Figure 6.3 shows the video of vehicle containing registration plate during rain environment. Rain is also a kind of noise. It is considered as salt and pepper noise. Figure 6.4 shows the extracted characters of the registration plate from the video of vehicle. 


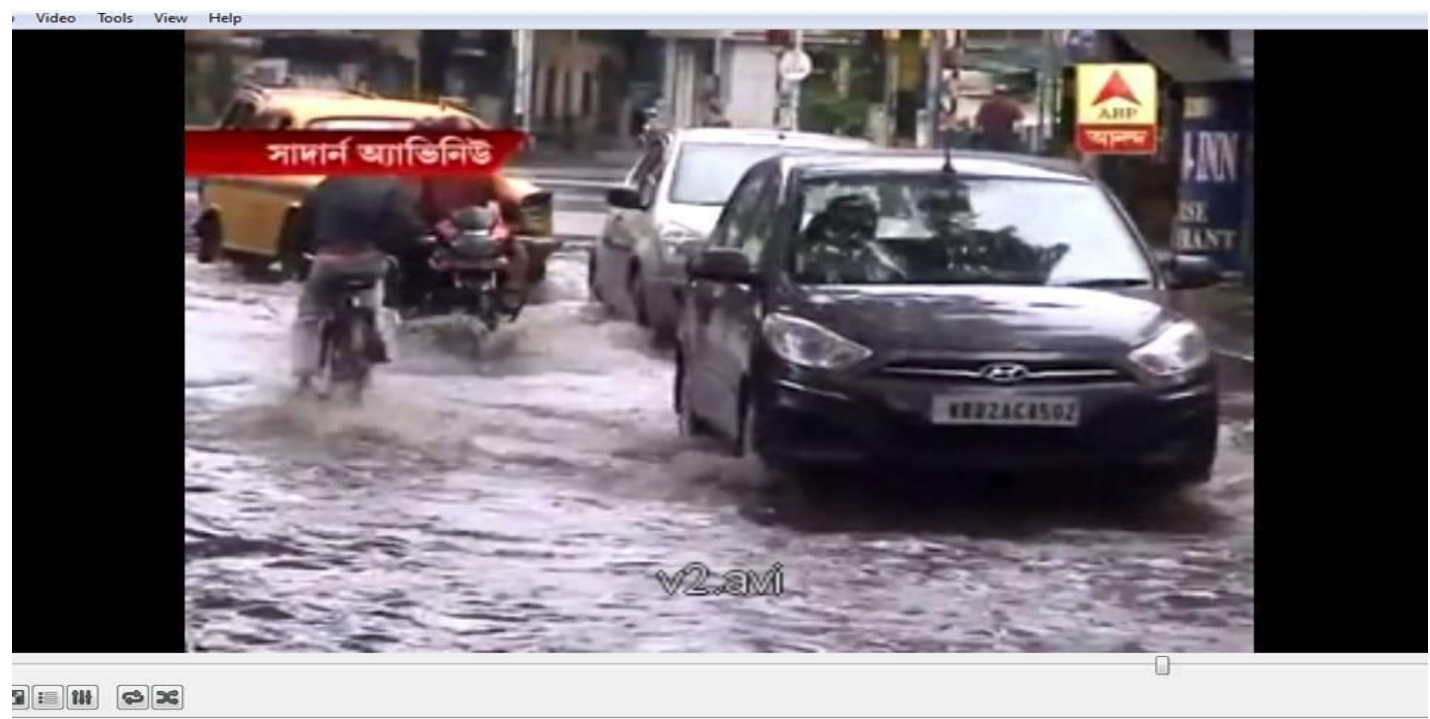

Figure 6.3: Video of vehicle containing Rain

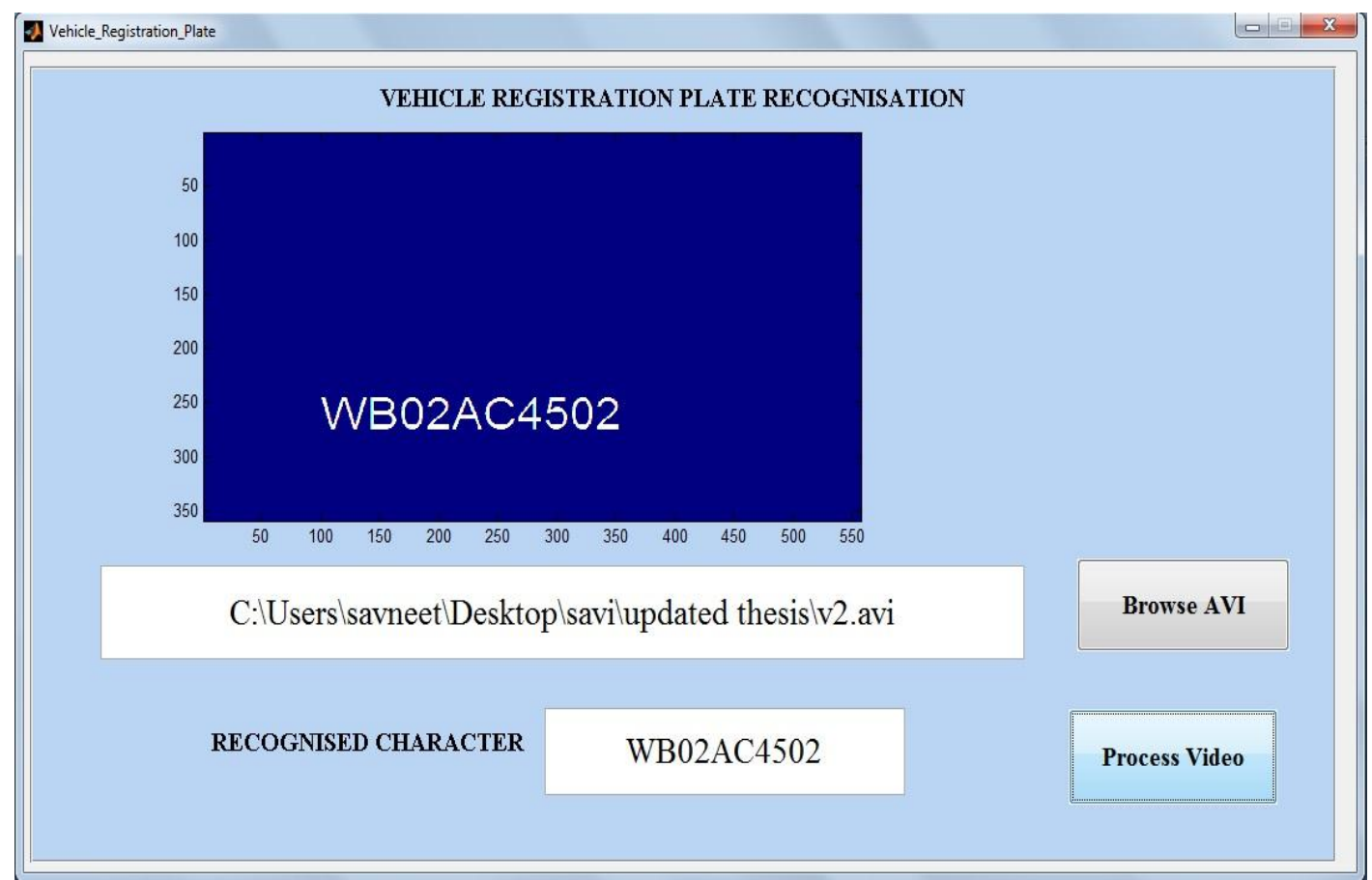

Figure 6.4: Successfully extracted registration plate image and characters from rain video.

\section{CONCLUSION \& FUTURE WORK}

It can be compiled from the above results that this technique is efficient in detecting and extracting registration plate of the vehicle, not only in clear environment but also in the noisy environment such as foggy and rainy. This can be helpful in areas such as traffic lights, petrol station, border areas and etc. In this technique not only image of registration late is extracted but the characters of the registration plate are also extracted. The future work for this technique is to automatically detect the faulty vehicle by using captured videos.

\section{REFERENCES}

[1] Abolghasemi, V. (2009), "An edge-based color-aided method for license plate detection", Image and Vision Computing, Vol. 27, No. 2, pp. 1134-1142.

[2] Ellis, J.S., and Penny, R.W. (2012), "Real time vehicle identification performance using FPGA correlation hardware", IEEE transaction on intelligent transportation system, Vol. 13, No. 4, pp. 1891-1895.

[3] Hung, K.M., and Hsieh, C.T. (2010), "A real time mobile vehicle licence plate detection and recognition", Tamkang journal of science and engineering, Vol. 13, No. 4, pp. 433-442. 
[4] Kaur, H., and Manvi (2012), "Vehicle Licence Plate Detection from video using edge detection and Morphological operators", International Journal of Engineering Research \& Technology (IJERT), Vol. 1, No. 9, pp. 1-5.

[5] Lazrus, A., and Choubey, S. (2011), "An efficient method of vehicle number plate detection and recognition", International journal of machine intelligence, Vol. 3, No. 3, pp. 134- 137.

[6] Martin, D.F., and Garcia, M.B. (2012), "Vehicular traffic surveillance and road lane detection using radar interferometry", IEEE transactions on vehicular technology, Vol. 61, No. 3, pp. 959-970.

[7] Paunwala, C. (2012), "A Novel Multiple License Plate Extraction Technique for Complex Background in Indian
Traffic Conditions", International Journal of Image Processing, Computer Science, Vol. 4, No. 2, pp. 106118.

[8] Soon, C.K., and Lin, K.C. (2012), "Malaysian car number plate detection and recognition system", Australian journal of basic and applied science, pp. 4959.

[9] Sreedhar, K., and Pantal, B. (2012), "Enhancement of image using morphological transformations", International journal of computer science and information technology, Vol. 4, No. 1, pp. 33-50.

[10] Sharma, P., and Sapra, G. (2013), "Computational algorithm for automatic recognition of vehicle registration plates", International journal of engineering and computer science, Vol. 2, No. 8, pp. 2412-2414. 